\title{
Antenatal cough-induced rib fractures: an uncommon cause of upper abdominal pain
}

\section{Balaba K $\mathbf{1}^{1}$}

\begin{abstract}
Rib fractures secondary to cough are an uncommon cause of thoracic or abdominal pain in pregnancy. There is little in the literature describing this accidental phenomenon during pregnancy and why fractures occur so easily. In most cases, they are diagnosed fortuitously during the course of investigation for other more common causes of acute chest and upper abdominal pain. We report the case of a 38 year old, G11P5 who was 35 weeks pregnant, admitted with sudden onset right upper quadrant and right sided chest pain post coughing. This pain was caused by a 10th right rib fracture which was managed conservatively with analgesia. Despite numerous reviews, the fracture was not diagnosed. The patient also suffered a rib fracture on the contralateral side in a previous pregnancy.
\end{abstract}

\section{INTRODUCTION}

$\mathrm{Rib}$ fractures due to cough alone or with an added factor of shearing stress have been recognized for many years. In the literature however, there remain only a few reports of this accident occurring in the antenatal period. Most obstetricians usually diagnose this condition as an inadvertent finding in the course of investigations. A direct relationship between pregnancy and rib fractures is yet to be demonstrated ${ }^{1}$.

The shape of the pregnant thorax, higher intrathoracic pressures of pregnancy, nutritional state and drugs may contribute to the pathophysiology of cough rib fractures. We present a case of a 38 year old in week 35 of her pregnancy with subsequent rib fractures presenting acutely as lower right sided chest pain and right upper quadrant abdominal pain.

${ }^{l}$ Principal House Officer in Obstetrics and Gynecology, Department of Obstetrics \& Gynaecology, Logan Hospital, Meadowbrook, QLD 4131, Australia

Correspondence: Dr. Kristina Balaba BSc MBBS \& Masters of Women's Health with Excellence (UNSW).

E-mail: kristina.balaba@health.qld.gov.au

(D) http://orcid.org/0000-0003-1994-8857

Competing interests: The authors report no conflict of interest

\section{CASE PRESENTATION}

A 38 year old, G11P5, presented to the Maternity Assessment Unit (MAU) at 35 weeks following two presentations to the Emergency Department (ED) earlier that day with sudden onset chest pain post cough. On assessment in the ED, right sided chest wall tenderness was noted however the patient denied any history of trauma. A chest x-ray was performed and was deemed unremarkable by the ED and no cause of pain was found. Subsequently the patient was discharged home.

The patient presented again later that day with complaints of ongoing chest pain and difficulty breathing. Repeat assessment in the emergency department suspected pneumonia as the cause of the patient's symptoms, She was treated accordingly and again discharged home. She subsequently presented to the MAU with complaints of decreased foetal movements as well as ongoing chest pain and upper abdominal pain. The pain was cyclical and unrelated to her breathing. She denied any history of PV loss or symptoms suggestive of pre-eclampsia.

Further questioning revealed a two-week history of non-productive cough with associated constant epigastric, right-sided sharp flank and shoulder pain. She denied any urinary symptoms and maintained a normal diet. She had a history of marijuana and cigarette consumption but denied the use of other illicit substances. Her past medical history was significant for a previous caesarean section at 31 weeks for uterine abruption. Whilst chest $\mathrm{x}$-ray was reported as normal on multiple occasions, review of the images on this occasion revealed a diagnosis of a displaced fracture of the 10th right rib as the cause of her pain (fig 1). On further questioning the patient had revealed that she had a rib fracture on the contralateral side in a previous pregnancy of which no cause was found. She was admitted for observation and analgesia. Blood tests and obstetric ultrasound were unremarkable.

The patient was management conservatively with consideration for chest wall strapping and cough suppressants give. The patient was counselled and encouraged to cease smoking.

During her admission, the patient complained of ongoing constant lower abdominal pain. During that time, she had irregular uterine activity and a tender, tense uterus with no resting tone. Cardiotocography was abnormal and she was diagnosed clinically with abruption. Decision was made for a category 1 caesarean for suspected abruption.

\section{DISCUSSION}

Rib fractures in pregnancy usually occur due to mechanical causes, as seen in the case presented, however consideration should also be given to metabolic causes. The shape of the pregnant thorax, higher intrathoracic pressures of pregnancy, nutritional state and potential drug use may have all contributed to the recurrence of rib fractures in this patient.

There are a few proposed mechanisms as to why cough-induced rib fractures occur more readily in pregnancy. In the pregnant patient, the capacity of the lower ribs to flare at any lung volume is reduced 


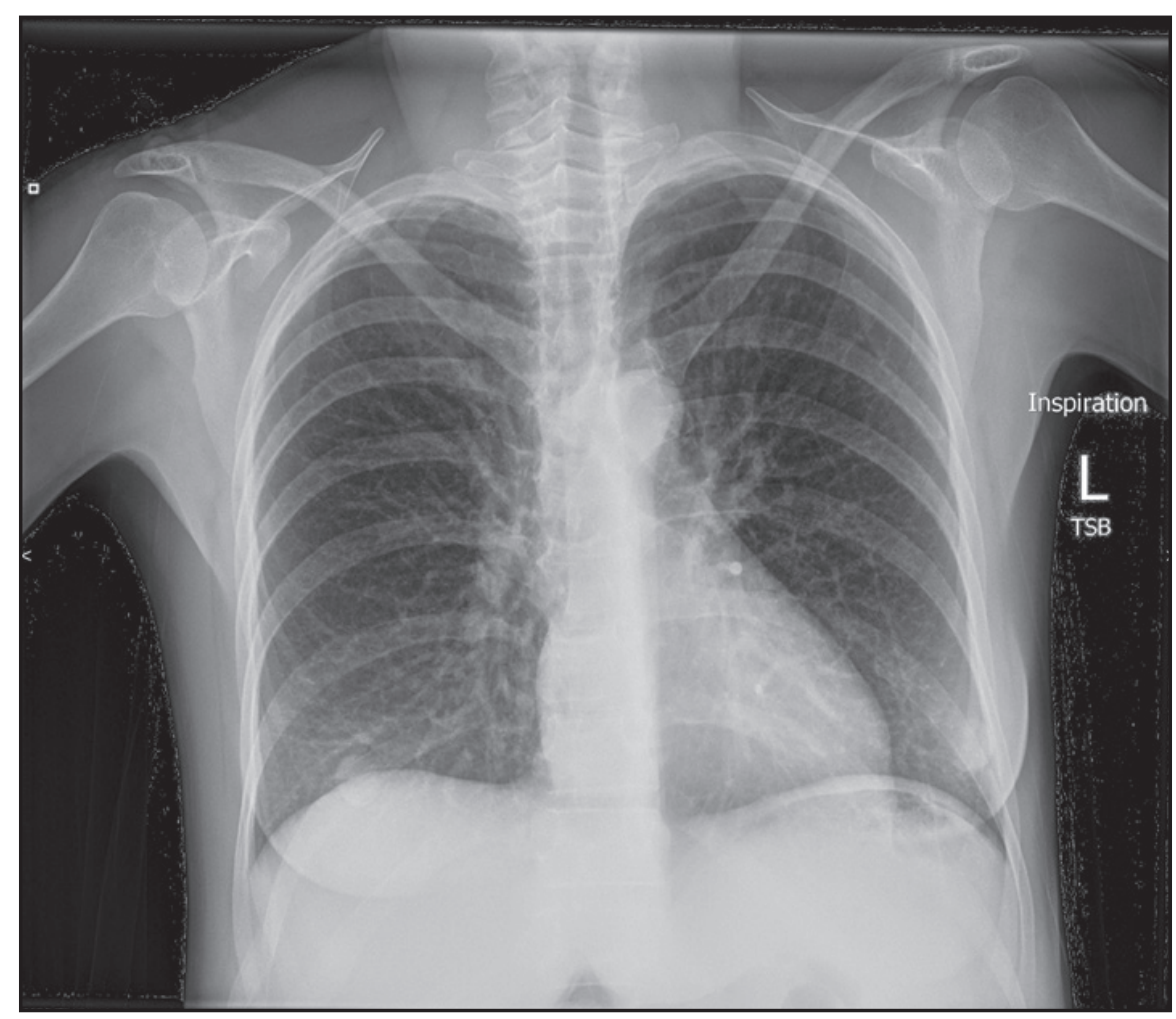

Figure 1 Chest radiograph showing displaced fracture of the 10th right rib.

as there is already disproportionately greater rib cage displacement due to the increased abdominal impedance. This may be the reason why the lower ribs are most likely to fracture under the influence of enormous bending forces that are applied at the height of a cough. The maximum allowable shear stress on ribs/trabecular bone, can be affected both by drugs and pregnancy. Although normal pregnancy ensures adequate delivery of minerals to the foetus whilst affording protection to the maternal skeleton, osteopenia from drugs and steroids may also contribute.

Looking at the fracture sites of many pregnancy cases, the shearing stress appears to occur along the line between the origin of the external oblique (from the lower four ribs) and the costal slips of the latissimus dorsi. Coughing in the pregnant state results in a compensatory lordosis from the head and shoulders being thrown back during the cough. Postural forces and possible hypertrophy of the iliocostalis and longisimus portion of the sacrospinalis with possible incoordination of action may also be involved. ${ }^{[2]}$

Another possible mechanism is the contraction of the anterior abdominal muscles during the cough which forces the uterine mass to be driven backwards and upwards against the inner surface of the lower chest. Thereby imparting a greater force resulting in fractures of the lower ribs. ${ }^{[3]}$

There is little in the literature looking at the effects of coughing in the pregnant patient, how the anatomical changes affect this and why fractures are more readily sustained during pregnancy. This may be an avenue for future research. Although fractured ribs may seem a trivial injury to some, it is one that can produce extreme discomfort and insomnia particularly in the obstetric patient and clinicians should keep such a diagnosis in mind when exposed to these patients.

\section{REFERENCES}

1. Baitner AC, Bernstein AD, Jazrawi AJ, Della Valle CJ, Jazrawi LM. Spontaneous rib fracture during pregnancy. A case report and review of the literature. Bull Hosp Jt Dis [Internet]. 2000;59(3):163-165.

2. Paulley JW, Lees DH, Pearson AC. Cough fracture in late pregnancy. Br Med J [Internet]. 1949 Jan; 1(4594):135-137.

3. Boyle RK. Cough stress rib fractures in two obstetric patients: case report and pathophysiology. Int $\mathrm{J}$ Obstet Anesth [Internet]. 1998 Jan;7(1):5458. 